\title{
The pLoc_bal-mPlant is a powerful artificial intelligence tool for predicting the subcellular localization of plant proteins purely based on their sequence information
}

\author{
Kuo-Chen Chou* \\ Gordon Life Science Institute, Boston, Massachusetts 02478, United States of America
}

Recently a very useful web-server, or AI (Artificial Intelligence) tool, has been developed for predicting the subcellular localization of plant proteins purely according to their information for the multi-label systems [1], in which a same protein may appear or travel between two or more locations and hence its ID (identification) needs two or more labels as well, namely the "multi-label mark" [2].

The AI tool is named as "pLoc_bal-mPlant", where "bal" stands for that the AI tool has been treated by balancing out the training dataset [3-9], and " $m$ " for that the AI tool can be used to investigate into the multi-label systems. Below, let us show how the AI tool is working.

Clicking the link at http://www.jci-bioinfo.cn/pLoc_bal-mPlant/, you will see the top page of the pLoc_bal-mPlant web-server prompted on your computer screen (Figure 1). Click the Example window and use the query protein sequences there as input and followed by clicking the Submit button, you will see Figure 2 on the screen of your computer. The corresponding detailed predicted results were given in ref.8. You can see from there: nearly all the success rates achieved by the AI tool for the plant proteins in each of the 12 subcellular locations are within the range of 97-100\%. Such a high prediction quality is far beyond the reach of any of its counterparts. In addition to the advantages of high accuracy and easy to use, the AI tool has been constructed by strictly complying with the "Chou's 5 -steps rule" and hence possesses the following terrific merits as concurred by

pLoc_bal-mPlant: predict subcellular localization of plant proteins by balancing training dataset and general PseAAC | Read Me | Supporting information | Citation |

Enter query sequences

Enter the sequences of query proteins in FASTA format (Example): the number of proteins is limited at 10 or less for each submission.

\section{Submit Cancel}

\section{Or, upload a file for batch prediction}

Enter your e-mail address and upload the batch input file (Batch-example).

The predicted result will be sent to you by e-mail once completed; it

usually takes 1 minute or so for each protein sequence.

Upload file: $\square$ Browse

Your Email:

Batch submit Cancel

Figure 1. A semi screenshot for the top page of pLoc_bal-mPlant (Adapted from [8] with permission)
Covered by pLoc_bal-mPlant are the following 12 subcellular locations
(1) Cell membrane
(2) Cell wall
(3) Chloroplast
(5) Endoplasmic reticulum
(7) Golgi apparatus
(9) Nucleus
(11) Plastid
(4) Cytoplasm
(6) Extracellular
(8) Mitochondrion
(10) Peroxisome
(12) Vacuole

\section{Predicted results}

\begin{tabular}{|c|c|}
\hline Protein ID & Subcellular location or locations \\
\hline$>004130$ & 3 \\
\hline$>P 15290$ & 2,4 \\
\hline$>P 54609$ & $2,4,9$ \\
\hline & Continue Test \\
\hline
\end{tabular}

Figure 2. A semi screenshot for the webpage obtained by following Step 3 of Section 3.5 (Adapted from [8] with permission)

many investigators (see, e.g., [10-91] as well as three comprehensive review papers $[2,92,93]):(1)$ crystal clear in logic development, (2) completely transparent in operation, (3) easily to repeat the reported results by other investigators, (4) with high potential in stimulating other sequence-analyzing methods, and (5) very convenient to be used by the majority of experimental scientists.

For the wonderful and awesome roles of the " 5 -steps rule" in driving proteome, genome analyses and drug development, see a series of recent papers [2,93-104] where the rule and its wide applications have been very impressively presented from various aspects or at different angles.

\section{References}

1. K.C. Chou, H.B. Shen, Recent progresses in protein subcellular location prediction. Analytical Biochemistry 370 (2007) 1-16.

${ }^{*}$ Correspondence to: Kuo-Chen Chou, Gordon Life Science Institute, Boston, Massachusetts 02478, United States of America, E-mail: kcchou@ gordonlifescience.org; kcchou38@gmail.com

Received: December 02, 2019; Accepted: December 21, 2019; Published: December 26, 2019 
Kuo-Chen C (2019) The pLoc_bal-mPlant is a powerful artificial intelligence tool for predicting the subcellular localization of plant proteins purely based on their sequence information

2. K.C. Chou, Advance in predicting subcellular localization of multi-label proteins and its implication for developing multi-target drugs. Current Medicinal Chemistry 26 (2019) 4918-4943.

3. X. Xiao, X. Cheng, G. Chen, Q. Mao, K.C. Chou, pLoc_bal-mVirus: Predict Subcellular Localization of Multi-Label Virus Proteins by Chou's General PseAAC and IHTS Treatment to Balance Training Dataset. Med Chem 15 (2019) 496-509.

4. X. Xiao, X. Cheng, G. Chen, Q. Mao, K.C.Chou, pLoc_bal-mGpos: predict subcellular localization of Gram-positive bacterial proteins by quasi-balancing training dataset and PseAAC. Genomics 111 (2019) 886-892.

5. K.C. Chou, X. Cheng, X. Xiao, pLoc_bal-mEuk: predict subcellular localization of eukaryotic proteins by general PseAAC and quasi-balancing training dataset. Med Chem 15 (2019) 472-485.

6. K.C. Chou, X. Cheng, X. Xiao, pLoc_bal-mHum: predict subcellular localization of human proteins by PseAAC and quasi-balancing training dataset Genomics 111 (2019) $1274-1282$

7. X. Cheng, W.Z. Lin, X. Xiao, K.C. Chou, pLoc bal-mAnimal: predict subcellular localization of animal proteins by balancing training dataset and PseAAC. Bioinformatics 35 (2019) 398-406.

8. X. Cheng, X. Xiao, K.C. Chou, pLoc bal-mPlant: predict subcellular localization of plant proteins by general PseAAC and balancing training dataset Curr Pharm Des 24 (2018) 4013-4022.

9. X. Cheng, X. Xiao, K.C. Chou, pLoc bal-mGneg: predict subcellular localization of Gram-negative bacterial proteins by quasi-balancing training dataset and general PseAAC. Journal of Theoretical Biology 458 (2018) 92-102.

10. A.H. Butt, Y.D. Khan, Prediction of S-Sulfenylation Sites Using Statistical Moments Based Features via Chou's 5-Step Rule. International Journal of Peptide Research and Therapeutics (IJPRT) https://doi.org/10.1007/s10989-019-09931-2 (2018).

11. M. Awais, W. Hussain, Y.D. Khan, N. Rasool, S.A. Khan, K.C. Chou, iPhosH-PseAAC: Identify phosphohistidine sites in proteins by blending statistical moments and position relative features according to the Chou's 5-step rule and general pseudo amino acid composition. IEEE/ACM Trans Comput Biol Bioinform https://doi.org/10.1109/ TCBB.2019.2919025 or https://www.ncbi.nlm.nih.gov/pubmed/31144645 (2019).

12. O. Barukab, Y.D. Khan, S.A. Khan, K.C. Chou, iSulfoTyr-PseAAC: Identify tyrosine sulfation sites by incorporating statistical moments via Chou's 5-steps rule and pseudo components Current Genomics https:/doi.org/10.2174/1389202920666190819091609 or http://www.eurekaselect.com/174277/article (2019).

13. A.H. Butt, Y.D. Khan, Prediction of S-Sulfenylation Sites Using Statistical Moments Based Features via Chou's 5-Step Rule. International Journal of Peptide Research and Therapeutics (IJPRT) htpps://doi.org/10.1007/s10989-019-09931-2 (2019).

14. Y. Chen, X. Fan, Use of Chou's 5-Steps Rule to Reveal Active Compound and Mechanism of Shuangshen Pingfei San on Idiopathic Pulmonary Fibrosis. Current Molecular Medicine https://doi.org/10.2174/1566524019666191011160543 (2019).

15. X. Du, Y. Diao, H. Liu, S. Li, MsDBP: Exploring DNA-binding Proteins by Integrating Multi-scale Sequence Information via Chou's 5-steps Rule. Journal of Proteome Research 18 (2019) 3119-3132.

16. Ehsan, M.K. Mahmood, Y.D. Khan, O.M. Barukab, S.A. Khan, K.C. Chou, iHydPseAAC (EPSV): Identify hydroxylation sites in proteins by extracting enhanced position and sequence variant feature via Chou's 5-step rule and general pseudo amino acid composition. Current Genomics 20 (2019) 124-133.

17. W. Hussain, S.D. Khan, N. Rasool, S.A. Khan, K.C. Chou, SPalmitoylC-PseAAC: A sequence-based model developed via Chou's 5-steps rule and general PseAAC for identifying S-palmitoylation sites in proteins. Anal Biochem 568 (2019) 14-23.

18. W. Hussain, Y.D. Khan, N. Rasool, S.A. Khan, K.C. Chou, SPrenylC-PseAAC: A sequence-based model developed via Chou's 5-steps rule and general PseAAC for identifying S-prenylation sites in proteins. J Theor Biol 468 (2019) 1-11.

19. Z. Ju, S.Y. Wang, Prediction of lysine formylation sites using the composition of k-spaced amino acid pairs via Chou's 5-steps rule and general pseudo components. Genomics htpps:/doi.org/10.1016/j.ygeno.2019.05.027 or https://www.ncbi.nlm.nih. gov/pubmed/31175975 (2019)

20. M. Kabir, S. Ahmad, M. Iqbal, M. Hayat, iNR-2L: A two-level sequence-based predictor developed via Chou's 5-steps rule and general PseAAC for identifying nuclear receptors and their families. Genomics https://www.ncbi.nlm.nih.gov/ pubmed/30779939 (2019).
21. Z.U. Khan, F. Ali, I.A. Khan, Y. Hussain, D. Pi, iRSpot-SPI: Deep learning-based recombination spots prediction byincorporating secondary sequence information coupled withphysio-chemical properties via Chou's 5-step rule and pseudo components. Chemometrics and Intelligent Laboratory Systems (CHEMOLAB) 189 (2019) 169 180 .

22. J. Lan, J. Liu, C. Liao, D.J. Merkler, Q. Han, J. Li, A Study for Therapeutic Treatment against Parkinson's Disease via Chou's 5-steps Rule. Current Topics in Medicinal Chemistry https://doi.org/10.2174/1568026619666191019111528 or http://www. eurekaselect.com/175887/article (2019).

23. N.Q.K. Le, iN6-methylat (5-step): identifying DNA N(6)-methyladenine sites in rice genome using continuous bag of nucleobases via Chou's 5-step rule. Mol Genet Genomics 294 (2019) 1173-1182.

24. N.Q.K. Le, E.K.Y. Yapp, Q.T. Ho, N. Nagasundaram, Y.Y. Ou, H.Y. Yeh, iEnhancer5Step: Identifying enhancers using hidden information of DNA sequences via Chou's 5-step rule and word embedding. Anal Biochem 571 (2019) 53-61.

25. N.Q.K. Le, E.K.Y. Yapp, Y.Y. Ou, H.Y. Yeh, iMotor-CNN: Identifying molecular functions of cytoskeleton motor proteins using $2 \mathrm{D}$ convolutional neural network via Chou's 5-step rule. Anal Biochem 575 (2019) 17-26.

26. R. Liang, J. Xie, C. Zhang, M. Zhang, H. Huang, H. Huo, X. Cao, B. Niu, Identifying Cancer Targets Based on Machine Learning Methods via Chou's 5-steps Rule and General Pseudo Components. Current Topics in Medicnal Chemistry htpps://doi.org/1 $0.2174 / 1568026619666191016155543$ (2019).

27. Y. Liang, S. Zhang, Identifying DNase I hypersensitive sites using multi-feature fusion and F-score features selection via Chou's 5-steps rule. Biophys Chem 253 (2019) 106227.

28. S.J. Malebary, M.S.U. Rehman, Y.D. Khan, iCrotoK-PseAAC: Identify lysine crotonylation sites by blending position relative statistical features according to the Chou's 5-step rule. PLoS One 14 (2019) e0223993.

29. Nazari, M. Tahir, H. Tayari, K.T. Chong, iN6-Methyl (5-step): Identifying RNA N6 methyladenosine sites using deep learning mode via Chou's 5-step rules and Chou's general PseKNC. Chemometrics and Intelligent Laboratory Systems (CHEMOLAB) htpps://doi.org/10.1016/j.chemolab.2019.103811 (2019).

30. Q. Ning, Z. Ma, X. Zhao, dForml(KNN)-PseAAC: Detecting formylation sites from protein sequences using K-nearest neighbor algorithm via Chou's 5-step rule and pseudo components. J Theor Biol 470 (2019) 43-49.

31. Salman, M. Khan, N. Iqbal, T. Hussain, S. Afzal, K.C. Chou, A two-level computation model based on deep learning algorithm for identification of piRNA and their functions via Chou's 5-steps rule. International Journal of Peptide Research and Therapeutics (IJPRT) htpps:/doi.org/10.1007/s10989-019-09887-3 (2019).

32. M. Tahir, H. Tayara, K.T. Chong, iDNA6mA (5-step rule): Identification of DNA N6methyladenine sites in the rice genome by intelligent computational model via Chou's 5-step rule. CHEMOLAB 189 (2019) 96-101.

33. S. Vishnoi, P. Garg, P. Arora, Physicochemical n-Grams Tool: A tool for protein physicochemical descriptor generation via Chou's 5-steps rule. Chem Biol Drug Des htpps://doi.org/10.1111/cbdd.13617 or https://www.ncbi.nlm.nih.gov/ pubmed/31483930 (2019).

34. Wiktorowicz, A. Wit, A. Dziewierz, L. Rzeszutko, D. Dudek, P. Kleczynski, Calcium Pattern Assessment in Patients with Severe Aortic Stenosis Via the Chou's 5-Steps Rule. Current Pharmaceutical Design https:/doi.org/10.2174/13816128256661909301 01258 (2019).

35. L. Yang, Y. Lv, S. Wang, Q. Zhang, Y. Pan, D. Su, Q. Lu, Y. Zuo, Identifying FL11 subtype by characterizing tumor immune microenvironment in prostate adenocarcinoma via Chou's 5-steps rule. Genomics https://doi.org/10.1016/j.ygeno.2019.08.021 (2019)

36. L. Yang, Y. Lv, S. Wang, Q. Zhang, Y. Pan, D. Su, Q. Lu, Y. Zuo, Identifying FL11 subtype by characterizing tumor immune microenvironment in prostate adenocarcinoma via Chou's 5-steps rule. Genomics (2019).

37. Y.D. Khan, N. Amin, W. Hussain, N. Rasool, S.A. Khan, K.C. Chou, iProteasePseAAC(2L): A two-layer predictor for identifying proteases and their types using Chou's 5-step-rule and general PseAAC. Anal Biochem 588 (2020) 113477.

38. Y. Xu, J. Ding, L.Y. Wu, K.C. Chou, iSNO-PseAAC: Predict cysteine S-nitrosylation sites in proteins by incorporating position specific amino acid propensity into pseudo amino acid composition PLoS ONE 8 (2013) e55844.

39. Y. Xu, X.J. Shao, L.Y. Wu, N.Y. Deng, K.C. Chou, iSNO-AAPair: incorporating amino acid pairwise coupling into PseAAC for predicting cysteine S-nitrosylation sites in proteins. PeerJ 1 (2013) e171. 
Kuo-Chen C (2019) The pLoc_bal-mPlant is a powerful artificial intelligence tool for predicting the subcellular localization of plant proteins purely based on their sequence information

40. Y. Xu, X. Wen, X.J. Shao, N.Y. Deng, K.C. Chou, iHyd-PseAAC: Predicting hydroxyproline and hydroxylysine in proteins by incorporating dipeptide positionspecific propensity into pseudo amino acid composition. International Journal of Molecular Sciences (IJMS) 15 (2014) 7594-7610

41. Y. Xu, X. Wen, L.S. Wen, L.Y. Wu, N.Y. Deng, K.C. Chou, iNitro-Tyr: Prediction of nitrotyrosine sites in proteins with general pseudo amino acid composition. PLoS ONE 9 (2014) e105018.

42. Y. Xu, K.C. Chou, Recent progress in predicting posttranslational modification sites in proteins. Curr Top Med Chem 16 (2016) 591-603.

43. L.M. Liu, Y. Xu, K.C. Chou, iPGK-PseAAC: identify lysine phosphoglycerylation sites in proteins by incorporating four different tiers of amino acid pairwise coupling information into the general PseAAC. Med Chem 13 (2017) 552-559.

44. Y. Xu, C. Li, K.C. Chou, iPreny-PseAAC: identify C-terminal cysteine prenylation sites in proteins by incorporating two tiers of sequence couplings into PseAAC. Med Chem 13 (2017) 544-551.

45. L. Cai, C.L. Wan, L. He, S. Jong, K.C. Chou, Gestational influenza increases the risk of psychosis in adults. Medicinal Chemistry 11 (2015) 676-682.

46. J. Liu, J. Song, M.Y. Wang, L. He, L. Cai, K.C. Chou, Association of EGF rs4444903 and XPD rs13181 polymorphisms with cutaneous melanoma in Caucasians. Medicinal Chemistry 11 (2015) 551-559.

47. L. Cai, Y.H. Yang, L. He, K.C. Chou, Modulation of cytokine network in the comorbidity of schizophrenia and tuberculosis. Curr Top Med Chem 16 (2016) 655-665.

48. L. Cai, W. Yuan, Z. Zhang, L. He, K.C. Chou, In-depth comparison of somatic poin mutation callers based on different tumor next-generation sequencing depth data Scientific Reports 6 (2016) 36540.

49. Y. Zhu, Q.W. Cong, Y. Liu, C.L. Wan, T. Yu, G. He, L. He, L. Cai, K.C. Chou, Antithrombin is an importantly inhibitory role against blood clots. Curr Top Med Chem 16 (2016) 666-674.

50. Z.D. Zhang, K. Liang, K. Li, G.Q. Wang, K.W. Zhang, L. Cai, S.T. Zha, K.C. Chou, Chlorella vulgaris induces apoptosis of human non-small cell lung carcinoma (NSCLC) cells. Med Chem 13 (2017) 560-568.

51. L. Cai, T. Huang, J. Su, X. Zhang, W. Chen, F. Zhang, L. He, K.C. Chou, Implications of newly identified brain eQTL genes and their interactors in Schizophrenia. Molecular Therapy - Nucleic Acids 12 (2018) 433-442.

52. B. Niu, M. Zhang, P. Du, L. Jiang, R. Qin, Q. Su, F. Chen, D. Du, Y. Shu, K.C. Chou, Small molecular floribundiquinone B derived from medicinal plants inhibits acetylcholinesterase activity. Oncotarget 8 (2017) 57149-57162.

53. Q. Su, W. Lu, D. Du, F. Chen, B. Niu, K.C. Chou, Prediction of the aquatic toxicity of aromatic compounds to tetrahymena pyriformis through support vector regression. Oncotarget 8 (2017) 49359-49369.

54. Y. Lu, S. Wang, J. Wang, G. Zhou, Q. Zhang, X. Zhou, B. Niu, Q. Chen, K.C. Chou, An Epidemic Avian Influenza Prediction Model Based on Google Trends. Letters in Organic Chemistry 16 (2019) 303-310.

55. B. Niu, C. Liang, Y. Lu, M. Zhao, Q. Chen, Y. Zhang, L. Zheng, K.C. Chou, Glioma stages prediction based on machine learning algorithm combined with protein-protein interaction networks. Genomics doi: 10.1016/j.ygeno.2019.05.024Get (2019).

56. J. Jia, Z. Liu, X. Xiao, B. Liu, K.C. Chou, Identification of protein-protein binding sites by incorporating the physicochemical properties and stationary wavelet transforms into pseudo amino acid composition (iPPBS-PseAAC). J Biomol Struct Dyn (JBSD) 34 (2016) 1946-1961.

57. J. Jia, Z. Liu, X. Xiao, B. Liu, K.C. Chou, iSuc-PseOpt: Identifying lysine succinylation sites in proteins by incorporating sequence-coupling effects into pseudo components and optimizing imbalanced training dataset. Anal Biochem 497 (2016) 48-56.

58. J. Jia, Z. Liu, X. Xiao, B. Liu, K.C. Chou, pSuc-Lys: Predict lysine succinylation sites in proteins with PseAAC and ensemble random forest approach. Journal of Theoretical Biology 394 (2016) 223-230.

59. J. Jia, Z. Liu, X. Xiao, B. Liu, K.C. Chou, iCar-PseCp: identify carbonylation sites in proteins by Monto Carlo sampling and incorporating sequence coupled effects into general PseAAC. Oncotarget 7 (2016) 34558-34570.

60. J. Jia, Z. Liu, X. Xiao, B. Liu, K.C. Chou, iPPBS-Opt: A Sequence-Based Ensemble Classifier for Identifying Protein-Protein Binding Sites by Optimizing Imbalanced Training Datasets. Molecules 21 (2016) E95.

61. J. Jia, L. Zhang, Z. Liu, X. Xiao, K.C. Chou, pSumo-CD: Predicting sumoylation sites in proteins with covariance discriminant algorithm by incorporating sequence-coupled effects into general PseAAC. Bioinformatics 32 (2016) 3133-3141.
62. Z. Liu, X. Xiao, D.J. Yu, J. Jia, W.R. Qiu, K.C. Chou, pRNAm-PC: Predicting $\mathrm{N}$-methyladenosine sites in RNA sequences via physical-chemical properties. Anal Biochem 497 (2016) 60-67.

63. X. Xiao, H.X. Ye, Z. Liu, J.H. Jia, K.C. Chou, iROS-gPseKNC: predicting replication origin sites in DNA by incorporating dinucleotide position-specific propensity into general pseudo nucleotide composition. Oncotarget 7 (2016) 34180-34189.

64. W.R. Qiu, B.Q. Sun, X. Xiao, Z.C. Xu, J.H. Jia, K.C. Chou, iKcr-PseEns: Identify lysine crotonylation sites in histone proteins with pseudo components and ensemble classifier. Genomics 110 (2018) 239-246.

65. J. Jia, X. Li, W. Qiu, X. Xiao, K.C. Chou, iPPI-PseAAC(CGR): Identify proteinprotein interactions by incorporating chaos game representation into PseAAC. Journal of Theoretical Biology 460 (2019) 195-203.

66. W. Chen, H. Ding, P. Feng, H. Lin, K.C. Chou, iACP: a sequence-based tool for identifying anticancer peptides. Oncotarget 7 (2016) 16895-16909.

67. W. Chen, P. Feng, H. Ding, H. Lin, K.C. Chou, Using deformation energy to analyze nucleosome positioning in genomes. Genomics 107 (2016) 69-75.

68. W. Chen, H. Tang, J. Ye, H. Lin, K.C. Chou, iRNA-PseU: Identifying RNA pseudouridine sites Molecular Therapy - Nucleic Acids 5 (2016) e332.

69. C.J. Zhang, H. Tang, W.C. Li, H. Lin, W. Chen, K.C. Chou, iOri-Human: identify human origin of replication by incorporating dinucleotide physicochemical properties into pseudo nucleotide composition. Oncotarget 7 (2016) 69783-69793.

70. W. Chen, P. Feng, H. Yang, H. Ding, H. Lin, K.C. Chou, iRNA-AI: identifying the adenosine to inosine editing sites in RNA sequences. Oncotarget 8 (2017) 4208-4217.

71. P. Feng, H. Ding, H. Yang, W. Chen, H. Lin, K.C. Chou, iRNA-PseColl: Identifying the occurrence sites of different RNA modifications by incorporating collective effects of nucleotides into PseKNC. Molecular Therapy - Nucleic Acids 7 (2017) 155-163.

72. W. Chen, H. Ding, X. Zhou, H. Lin, K.C. Chou, iRNA(m6A)-PseDNC: Identifying N6methyladenosine sites using pseudo dinucleotide composition. Analytical Biochemistry 561-562 (2018) 59-65.

73. W. Chen, P. Feng, H. Yang, H. Ding, H. Lin, K.C. Chou, iRNA-3typeA: identifying 3-types of modification at RNA's adenosine sites. Molecular Therapy: Nucleic Acid 11 (2018) 468-474.

74. Z.D. Su, Y. Huang, Z.Y. Zhang, Y.W. Zhao, D. Wang, W. Chen, K.C. Chou, H. Lin, iLoc-lncRNA: predict the subcellular location of lncRNAs by incorporating octamer composition into general PseKNC. Bioinformatics 34 (2018) 4196-4204.

75. H. Yang, W.R. Qiu, G. Liu, F.B. Guo, W. Chen, K.C. Chou, H. Lin, iRSpot-Pse6NC Identifying recombination spots in Saccharomyces cerevisiae by incorporating hexamer composition into general PseKNC International Journal of Biological Sciences 14 (2018) 883-891

76. P. Feng, H. Yang, H. Ding, H. Lin, W. Chen, K.C. Chou, iDNA6mA-PseKNC: Identifying DNA $\mathrm{N}(6)$-methyladenosine sites by incorporating nucleotide physicochemical properties into PseKNC. Genomics 111 (2019) 96-102.

77. Q.S. Du, S.Q. Wang, N.Z. Xie, Q.Y. Wang, R.B. Huang, K.C. Chou, 2L-PCA: A twolevel principal component analyzer for quantitative drug design and its applications. Oncotarget 8 (2017) 70564-70578.

78. B. Liu, L. Fang, R. Long, X. Lan, K.C. Chou, iEnhancer-2L: a two-layer predicto for identifying enhancers and their strength by pseudo k-tuple nucleotide composition. Bioinformatics 32 (2016) 362-369.

79. B. Liu, R. Long, K.C. Chou, iDHS-EL: Identifying DNase I hypersensi-tivesites by fusing three different modes of pseudo nucleotide composition into an ensemble learning framework. Bioinformatics 32 (2016) 2411-2418.

80. B. Liu, S. Wang, R. Long, K.C. Chou, iRSpot-EL: identify recombination spots with an ensemble learning approach. Bioinformatics 33 (2017) 35-41.

81. B. Liu, F. Yang, K.C. Chou, 2L-piRNA: A two-layer ensemble classifier for identifying piwi-interacting RNAs and their function. Molecular Therapy - Nucleic Acids 7 (2017) 267-277.

82. B. Liu, K. Li, D.S. Huang, K.C. Chou, iEnhancer-EL: Identifying enhancers and their strength with ensemble learning approach. Bioinformatics 34 (2018) 3835-3842.

83. B. Liu, F. Weng, D.S. Huang, K.C. Chou, iRO-3wPseKNC: Identify DNA replication origins by three-window-based PseKNC. Bioinformatics 34 (2018) 3086-3093.

84. B. Liu, F. Yang, D.S. Huang, K.C. Chou, iPromoter-2L: a two-layer predictor for identifying promoters and their types by multi-window-based PseKNC. Bioinformatics 34 (2018) 33-40. 
Kuo-Chen C (2019) The pLoc_bal-mPlant is a powerful artificial intelligence tool for predicting the subcellular localization of plant proteins purely based on their sequence information

85. W.R. Qiu, B.Q. Sun, X. Xiao, Z.C. Xu, K.C. Chou, iHyd-PseCp: Identify hydroxyproline and hydroxylysine in proteins by incorporating sequence-coupled effects into general PseAAC. Oncotarget 7 (2016) 44310-44321.

86. W.R. Qiu, B.Q. Sun, X. Xiao, Z.C. Xu, K.C. Chou, iPTM-mLys: identifying multiple lysine PTM sites and their different types. Bioinformatics 32 (2016) 3116-3123.

87. W.R. Qiu, X. Xiao, Z.C. Xu, K.C. Chou, iPhos-PseEn: identifying phosphorylation sites in proteins by fusing different pseudo components into an ensemble classifier. Oncotarget 7 (2016) 51270-51283.

88. W.R. Qiu, S.Y. Jiang, B.Q. Sun, X. Xiao, X. Cheng, K.C. Chou, iRNA-2methyl: identify RNA 2'-O-methylation sites by incorporating sequence-coupled effects into general PseKNC and ensemble classifier. Medicinal Chemistry 13 (2017) 734-743.

89. W.R. Qiu, S.Y. Jiang, Z.C. Xu, X. Xiao, K.C. Chou, iRNAm5C-PseDNC: identifying RNA 5-methylcytosine sites by incorporating physical-chemical properties into pseudo dinucleotide composition. Oncotarget 8 (2017) 41178-41188.

90. W.R. Qiu, B.Q. Sun, X. Xiao, D. Xu, K.C. Chou, iPhos-PseEvo: Identifying human phosphorylated proteins by incorporating evolutionary information into general PseAAC via grey system theory. Molecular Informatics 36 (2017) UNSP 1600010.

91. X. Zhai, M. Chen, W. Lu, Accelerated search for perovskite materials with higher Curie temperature based on the machine learning methods. Computational Materials Science 151 (2018) 41-48.

92. K.C. Chou, Some remarks on protein attribute prediction and pseudo amino acid composition (50th Anniversary Year Review, 5-steps rule). Journal of Theoretical Biology 273 (2011) 236-247.

93. K.C. Chou, Impacts of pseudo amino acid components and 5-steps rule to proteomics and proteome analysis. Current Topics in Medicinak Chemistry (CTMC) (Special Issue ed. G.P Zhou) https://doi.org/10.2174/1568026619666191018100141 or http://www. eurekaselect.com/175823/article (2019).

94. K.C. Chou, Two kinds of metrics for computational biology. Genomics https://www. sciencedirect.com/science/article/pii/S0888754319304604?via\%3Dihub (2019).
95. K.C. Chou, Proposing pseudo amino acid components is an important milestone for proteome and genome analyses. International Journal for Peptide Research and Therapeutics (IJPRT) https://doi.org/10.1007/s10989-019-09910-7 or https://link. springer.com/article/10.1007\%2Fs10989-019-09910-7 (2019).

96. K.C. Chou, An insightful recollection for predicting protein subcellular locations in multi-label systems. Genomics http://doi.org/10.1016/j.ygeno.2019.08.008 or https:// www.sciencedirect.com/science/article/pii/S0888754319304604?via\%3Dihub (2019).

97. K.C. Chou, Progresses in predicting post-translational modification. International Journal of Peptide Research and Therapeutics (IJPRT) https:/doi.org/10.1007/s10989-01909893-5 or https://link.springer.com/article/10.1007\%2Fs10989-019-09893-5 (2019).

98. K.C. Chou, Recent Progresses in Predicting Protein Subcellular Localization with Artificial Intelligence (AI) Tools Developed Via the 5-Steps Rule. Japanese Journal of Gastroenterology and Hepatology https:/doi.org/www.jjgastrohepto.org or https:// www.jjgastrohepto.org/ (2019).

99. K.C. Chou, An insightful recollection since the distorted key theory was born about 23 years ago. Genomics https://doi.org/10.1016/j.ygeno.2019.09.001 or https://www. sciencedirect.com/science/article/pii/S0888754319305543?via\%3Dihub (2019).

100. K.C. Chou, Artificial intelligence (AI) tools constructed via the 5-steps rule for predicting post-translational modifications. Trends in Artificial Inttelengence (TIA) 3 (2019) 60-74

101. K.C. Chou, Distorted Key Theory and Its Implication for Drug Development. Current Genomics http://www.eurekaselect.com/175823/article or http://www.eurekaselect. com/175823/article (2020).

102. Chou, K.C. An Insightful 10-year Recollection Since the Emergence of the 5-steps Rule. Current Pharmaceutical Design 2019, 25, 4223-4234.

103. K.C. Chou, An insightful recollection since the birth of Gordon Life Science Institute about 17 years ago. Advancement in Scientific and Engineering Research 4 (2019) 31-36.

104. K.C. Chou, Gordon Life Science Institute: Its philosophy, achievements, and perspective. Annals of Cancer Therapy and Pharmacology 2 (2019) 001-26.

Copyright: ( 92019 Kuo-Chen C. This is an open-access article distributed under the terms of the Creative Commons Attribution License, which permits unrestricted use, distribution, and reproduction in any medium, provided the original author and source are credited. 